\title{
ReaR

\section{Uso del ECG endocavitario en la canalización de vías centrales}

Clemente Armendáriz L, Casimiro Erviti A, Batllori Gastón M.

Complejo Hospitalario de Navarra.

\section{Resumen}

La canalización de vías centrales supone, hoy en día, una práctica habitual en el ámbito de la Anestesiología. Mediante este artículo queremos presentar el uso del ECG endocavitario como método alternativo para guiar la adecuada colocación del catéter. Se trata de una técnica útil, inocua y fácil de llevar a cabo. Acompañamos el texto de un vídeo explicativo para facilitar la comprensión de la técnica.

\section{Introducción}

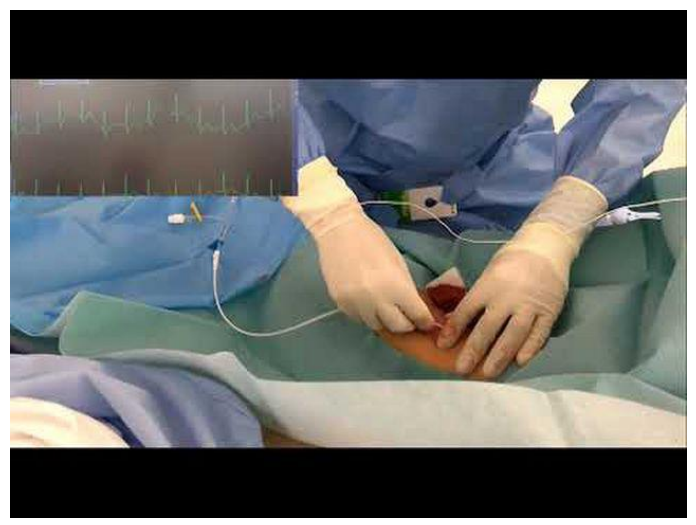

La canalización de vías centrales supone, hoy en día, una práctica habitual en el ámbito de la Anestesiología. Mediante este artículo queremos presentar el uso del ECG endocavitario como método alternativo para guiar la adecuada colocación del catéter. Se trata de una técnica útil, inocua y fácil de llevar a cabo. Acompañamos el texto de un vídeo explicativo para facilitar la comprensión de la técnica.

La implantación de la ecografía como método de asistencia para realizar las técnicas de acceso venoso central ha permitido optimizar la seguridad del paciente y disminuir la incidencia de complicaciones mecánicas.

Sin embargo, si bien la asistencia ecográfica facilita la canulación en sí misma, no es tan útil para verificar la adecuada posición de la punta del catéter. Tradicionalmente se han empleado medios radiológicos para verificar dicha posición, bien sea durante las propias maniobras de canulación (radioscopia) o bien después de las mismas (radiografía de tórax). La radioscopia permite verificar en tiempo real, durante la colocación del catéter, el correcto avance de este hacia la vena cava superior.

Existen otros medios para guiar la colocación del catéter en tiempo real, que permiten prescindir de la exposición radiológica. La verificación ecográfica del avance del catéter hacia la cava superior exige la visualización de esta, bien sea mediante ecografía transtorácica $o$ transesofágica. Este método demanda la presencia de un operador experimentado en ecografía para realizar la exploración y asistir al canulador, y además no es siempre 
aplicable (mala ventana ecocardiográfica transtorácica) ni está exento de complicaciones (ecografía transesofágica).

El registro ECG endocavitario es un método alternativo para guiar la colocación del catéter, y es el objeto del presente artículo.

\section{Descripción de la técnica}

Esta técnica, descrita inicialmente en 1949, se basa en la utilización de la guía de Seldinger (guía en J) como un electrodo ECG endovascular.

Pasos a seguir:

1- En primer lugar, se deben verificar las marcas del catéter y la guía en $\mathrm{J}$, comprobando la posición en la que la punta de la guía en $J$ sobresale por la punta del catéter.

2- Se procede a canular el vaso utilizando la técnica de Seldinger.

3- Una vez llegue el momento de avanzar el catéter sobre la guía en J, dicho avance debe realizarse hasta que la punta del catéter se encuentre alineada con la punta de la guía en $\mathrm{J}$.

4- Se utiliza un cable especial para pinzar la guía metálica en su extremo proximal y conectarla a un dispositivo que permitirá emplear la guía como electrodo del hombro derecho en el ECG. En nuestro caso empleamos un conmutador que permite utilizar alternativamente el electrodo del hombro derecho o la guía metálica.

5- Una vez conectado el cable al conmutador, se acciona un interruptor para pasar a utilizar la guía metálica como electrodo del hombro derecho. A continuación, se procede a analizar los cambios en la onda " $p$ " en la derivación II (hombro derecho - pierna izquierda).
A medida que el catéter avanza a través de la vena cava superior hacia la aurícula derecha se constatará que la onda " $p$ " se hace progresivamente más picuda según se aproxima al nodo sinusal, llegando a ser isodifásica cuando esté a la altura del mismo y haciéndose negativa cuando se avance más allá de él.

6- El conjunto catéter - guía metálica debe avanzarse hasta que se registre una altura máxima de onda "p". Esto indica que la punta del catéter está en la entrada de la aurícula derecha. Si se retira el conjunto catéter - guía metálica se observa una reducción gradual de la altura de la onda " $p$ ". Una vez que se observa que la altura de la onda "p" es aproximadamente la mitad de la altura máxima, esto significa que la punta del catéter está en el tercio inferior de la vena cava superior, justo por encima de la unión cavo-auricular. Si se prosigue la retirada del catéter, cuando la altura de la onda " $p$ " alcanza dimensiones "normales", esto indica que la punta está nuevamente en la parte superior de la vena cava superior.

Ventajas de la técnica de registro ECG endocavitario:

- Se trata de un método preciso. - Es fácil y rápido de realizar, sin incrementar significativamente la duración del procedimiento. - Es fácil de enseñar y fácil de aprender. - Es seguro para el paciente, evitando la exposición a radiaciones ionizantes. - Se minimiza el riesgo de arritmias iatrogénicas debidas a irritación mecánica de las paredes de la aurícula o ventrículo, puesto que se detecta cuando el catéter se acerca a la aurícula. - Puede ser utilizado en la gran mayoría de los pacientes candidatos para acceso venoso central. La única excepción son aquellas condiciones clínicas en las que la onda "p" no se puede reconocer en el ECG (fibrilación / flúter auricular). 
- Permite una comprobación en tiempo real de la posición de la punta durante el procedimiento, evitando recurrir a maniobras de reposicionamiento posteriores.

- Se puede aplicar en situaciones clínicas donde la verificación radiológica está contraindicada (embarazo) o es logísticamente difícil (inserción de PICC en un domicilio). - Es económico.

\section{Uso del ECG endocavitario en la canalización de vías centrales (ENLACE VÍDEO EN VIMEO AQUÍ)}

\section{Conclusiones}

La verificación de la posición correcta de la punta del catéter de vía central es de suma importancia, puesto que permite prevenir complicaciones (trombosis secundarias a malposición del catéter / catéteres "a contracorriente" en el sistema venoso, perforación cardíaca) y debe hacerse antes de comenzar a utilizar el catéter. Son preferibles los métodos intraprocedimentales para verificar la correcta ubicación ya que evitan riesgos, demoras y costes de reposicionamiento posterior. El uso del ECG endocavitario puede usarse aproximadamente en un $93 \%$ de los pacientes según algunas publicaciones. Además, podría eliminar la necesidad de una radiografía de tórax posterior al procedimiento, siempre y cuando no haya un riesgo de daño pleuro-pulmonar que se deba descartar.

\section{Bibliografía}

1. Pittiruti M, la Greca A and Scoppettuolo G. The electrocardiographic method for positioning the tip of central venous catheters. J Vasc Access 2011; 12(4): 280-291. (PubMed)

2. Electrocardiographic-guided tip positioning technique for peripherally inserted central catheters in a Dutch teaching hospital: Feasibility and cost-effectiveness análisis in a prospective cohort study. Bloemen A, Daniels AM, Samyn MG, Janssen RJ, Elshof JW. J Vasc Access. 2018 Mar 1:1129729818764051. (PubMed)

Correspondencia al autor

Luis Clemente Armendáriz

luisclemente160@gmail.com

FEA anestesia y reanimación.

Complejo Hospitalario de Navarra.

Aceptado para el blog en febrero de 2019. 